\title{
Polo-Alvarado, Lorna. Vida de mujeres: entre la sumisión y la subversión. San Juan: Editorial Luscinia C.E., 2018.
}

DOI: http://dx.doi.org/10.15648/hc.37.2020.11

Si la preocupación por la vida de mujeres no se ha convertido en una de las temáticas más importantes y fascinantes de los últimos tiempos en la academia hispanoamericana, al menos el texto de la investigadora Lorna Polo-Alvarado examina con bastante claridad la situación de la mujer en el pasado. La cultura popular e histórica, los legados de la misoginia y el chauvinismo occidental enraizado en la cultura y la literatura de todos los tiempos aparecen puntualmente en el análisis del libro en cuestión. Por lo tanto, la literatura de mujeres y las convenciones de género emergen en la narrativa de Vida de mujeres para causar interesantes conflictos en medio de la tradición masculinizante que ha plagado la formación de Occidente. Dicho lo anterior, cabe decir que ha existido una perenne dislocación de conceptos que sugieren la perpetuación de servilismo y esclavitud en el imaginario lingüístico y cultural de la familia y su respectiva asignación de roles de género.

La pregunta que nos convoca en esta reseña intenta aclarar si el texto de la investigadora Lorna Polo-Alvarado rompe con la conocida teoría feminista en poner a la "mujer por encima del hombre", apoyando así la crítica feminista radical, o si por el contrario, el análisis lo basa en un estudio mesurado e histórico de la contribución factual de la mujer en cada esfera de la sociedad circundante. Por lo tanto, mi intención con esta reseña es responder a los siguientes cuestionamientos, a saber: ¿En qué medida este nuevo libro sobre mujeres aporta a la academia y a las investigaciones de género? ¿Cuál es la aproximación literaria e historicista que se utiliza en la narrativa del libro a analizar? ¿De qué manera Vida de mujeres capacita a los intelectuales actuales a reaccionar en 
contra del pasado oscuro en el que se lanzó a la mujer, y más bien, procura la reivindicación total de la misma hoy en día?

Una de las fortalezas del presente libro es la aproximación interdisciplinaria y sistemática al rastrear los cambios (si ha habido algunos) de la vida de mujeres desde la época clásica hasta su participación en las ciencias. La autora elabora un exitoso recorrido "demarcando la realidad de la mujer en la España medieval"", y presta particular atención a los siglos XVI y XVII. El libro es soportado por una variedad de análisis donde la autora identifica factores que constituyen datos explícitos y verídicos extraídos de fuentes históricas fidedignas. Así, dicho texto analiza la vida de mujeres en la rama política, religiosa, social y en el seno familiar igualmente, como indicadores sustanciales de una construcción de un discurso histórico elaborado por los poderes de turno.

En la introducción del libro, Polo-Alvarado busca explicar la amalgama de tradiciones femeninas de la cultura occidental en los períodos correspondientes. Igualmente, la autora argumenta que se ha "seleccionado una diversidad de fuentes que van desde textos jurídicos, históricos, médicos, literarios y religiosos de cada época hasta los estudios contemporáneos sobre historia, derecho, crítica literaria, arte, sociología, economía, feminismo, entre otros"2. Polo-Alvarado se enfoca primordialmente en el papel de la mujer desde la familia y su entorno, lugar asignado por la sociedad para funcionar y supuestamente desarrollarse.

Después de elaborar un corto epígrafe sobre la misoginia versus defensa de la mujer, en el cual describe las cualidades religiosas recurrentes en el texto bíblico, la profesora Polo-Alvarado divide su libro en un corpus sustancioso de siete capítulos que se expanden desde la época clásica (Grecia y Roma), Edad Media, Renacimiento y Barroco españoles, incluyendo igualmente la vida monástica femenina y el desenvolvimiento de la mujer en la vida cotidiana y económica, finalizando con su participación en las ciencias.

1 Lorna Polo-Alvarado, Vida de mujeres: entre la sumisión y la subversión (San Juan: Editorial Luscinia C.E., 2018), 11.

2 Lorna Polo-Alvarado, Vida de mujeres: entre la sumisión, 11. 
El primer capítulo, titulado "Época clásica (Grecia y Roma)", adecuadamente trata de la situación de la mujer durante la época clásica prestando atención a Grecia y a Roma. Como bien lo explica la autora, "las fuentes utilizadas [fueron] la historia y la literatura que produjeron estas

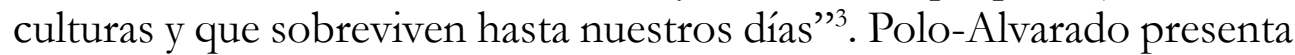
un sugestivo análisis sobre la vida femenina en estas dos culturas enfatizando su rol dentro del matrimonio e igualmente las mujeres en su vida cortesana. Específicamente causa gran interés la apropiación que la familia del marido creaba en la esposa romana, lo que se determina como cum manu. Es decir, al desposarse la mujer pasaba a ser propiedad de la familia del esposo perdiendo así los derechos como individuo independiente. Al parecer existía una tradición clandestina en la que la mujer era dueña del divorcio y también de la posibilidad del aborto a pesar de que "no siempre fuera por decisión propia".

Mientras el primer capítulo se enfoca en Grecia y Roma, el capítulo dos estudia el estatus social de la mujer en la complejidad de la Edad Media. La autora presenta evidencias de la labor académica femenina sobre todo en la poesía. Algo interesante en el presente capítulo es el valioso aporte de la profesora Polo-Alvarado en cuanto a la España musulmana. Según esta autora e igualmente retomando algunos críticos importantes, [las mujeres de la España musulmana] tuvieron tantas o más posibilidades que sus contemporáneas cristianas ${ }^{5}$. Es decir, Polo-Alvarado trastoca un poco lo que siempre se ha dicho del veto de la mujer en la Antigüedad y enfatiza que "la presencia pública de mujeres en fiestas no era solo en el caso de la prostitución puesto que las mujeres "decentes" tenían también acceso a estas diversiones, aunque habría que preguntarse en cuál grado y con cuánta frecuencia" . En resumidas, la investigadora argumenta que la vida de las mujeres no solamente se vio restringida en su totalidad, sino que más bien tenía que ver con la clase social en la que se desenvolvían y por lo tanto, no podemos comparar la vida de las esclavas o las campesinas con la de las mujeres de la nobleza, ya que no vivían bajo las mismas circunstancias. 
Por otro lado, tanto el capítulo tres "Renacimiento y Barroco españoles" como el capítulo cuatro "Mujeres en la vida religiosa, monjas, beguinas y beatas" claramente analizan la vida de las mujeres en los varios núcleos institucionalizados como la vida matrimonial y la vida eclesial. Aunque todavía la realidad social de las mujeres de los siglos en cuestión continuaba siendo inferior, varios cambios se dieron en los siglos posteriores. Uno de esos cambios fue la vida monástica de aquellas mujeres que optaban por no casarse. El capítulo de vida conventual femenina ofrece un análisis profundo de las complejidades de la vida diaria en la vida religiosa durante la Edad Media hasta la España del siglo XVII. Polo-Alvarado explica con detalle las marcadas diferencias entre monjas, beatas y beguinas, todas con un acerbo fiel a la fe cristiana, pero también con suficiente resentimiento e inclinaciones de poder religioso y social para rebeldizarse contra la Iglesia, en especial, los clérigos que imponían sus dogmas.

Los últimos tres capítulos reflejan la visión general de las mujeres en la delincuencia, la economía y en las respectivas ciencias. Uno de los capítulos involucra la relación con la historia de mujeres que asumen la delincuencia como manera de emancipación o se convierten en asesinas debido a la opresión durante el período en cuestión. En el capítulo cinco, "Las mujeres y la delincuencia", Polo-Alvarado ofrece un estudio pormenorizado de la situación de las mujeres delincuentes al igual que el proceso de victimización de algunas de ellas por parte de las instituciones gubernamentales y religiosas. Uno de los delitos en cuestión era el delito sexual reflejado en actos "contra natura", como el lesbianismo, el cual "preferían silenciarlo y obviarlo" . De allí que -como explica Polo-Alvarado- "sus consecuencias judiciales son raras en la documentación histórica que normalmente encuentran los investigadores"

Este capítulo se hace interesante puesto que la investigadora plantea “juzgar los casos [...] a la luz de la vida según los siglos aquí atendidos, y [...] no juzgarles según la mentalidad o realidad de[1] siglo XXI"”. A

7 Lorna Polo-Alvarado, Vida de mujeres: entre la sumisión, 95.

8 Lorna Polo-Alvarado, Vida de mujeres: entre la sumisión, 95.

9 Lorna Polo-Alvarado, Vida de mujeres: entre la sumisión, 94. 
mi parecer la autora salvaguarda la presión que ejercen algunos críticos de hoy día para analizar relatos o situaciones históricas según los ojos contemporáneos olvidándose que los hechos históricos son propios de su época. No con lo anterior creo dar el aval para apoyar atrocidades o genocidios o aún discriminación contra el ser humano en general, pero sí pienso que la crítica literaria y las teorías deben tener en cuenta el contexto histórico del hecho a analizar. Es decir, el investigador contemporáneo -como en el caso de la profesora Lorna Polo-Alvaradodebe analizar el contexto histórico desde el mismo contexto situacional, entendiendo la problemática social, religiosa y económica en la que se enmarcaban los delitos y conflictos de la época a analizar. Lo anterior la profesora Polo-Alvarado lo maneja con gran maestría y dedicación.

El capítulo seis "Las mujeres en la economía" es escrito con destreza puesto que inicia con las labores de la mujer en casa con los oficios domésticos y la crianza de sus hijos, al igual que la cocina y los procesos de tejido e hilado. Es decir, los períodos históricos que la profesora Polo-Alvarado está analizando no se pueden entender sin la inclusión de la mujer en la economía, sea muchas veces asalariada o no. La economía y las finanzas de la mujer no solamente se basan en sus labores domésticas, como normalmente se ha mal entendido, sino también la mujer sale de casa a conseguir recursos para la subsistencia y manutención de su propia familia, muchas veces al lado del esposo. En algunas ocasiones compartía las labores con el esposo fuera de casa y ambos conseguían salario para traer al hogar.

Interesantemente Polo-Alvarado establece que una de las labores de la mujer para conseguir sustento durante la España de la Edad Media y el Siglo de Oro fue la prostitución. A dichas mujeres se les llamaba "prostitutas, cantoneras o "malas mujeres"'10. La autora continúa comentando que la prostitución se procesó en tres etapas: "la época en que era un oficio reglamentado y legal; la prohibida que experimentó gran expansión, y el período en que era castigada como delito y mal social" "11. Otro de los delitos penalizados era el de revendedoras de productos para

10 Lorna Polo-Alvarado, Vida de mujeres: entre la sumisión, 105.

11 Lorna Polo-Alvarado, Vida de mujeres: entre la sumisión, 105. 
sacar provecho o intereses a ciertos alimentos como las aves, los pescados, el pan y el vino. Cabe decir que la "la mayoría de las mujeres que ejercían oficios, por la necesidad de remuneración, eran campesinas y mujeres pobres"12.

Por otro lado, y si entendemos la vida de las mujeres solamente como labores domésticas, creo que se está perdiendo el meollo de todo el mundo femenino durante estos períodos de la historia en España. El pensamiento femenino se involucra no solamente en las labores de casa, sino también fuera de ella; dichas acciones de las mujeres crean relaciones de interdependencia y círculos sociales que entran en las narrativas y en los eventos de dicho contexto histórico. No necesariamente -como comenta Polo-Alvarado en algunos apartes- se tiene que comprender la vida de las mujeres desde la óptica contemporánea sino más bien entender el suceso en su contexto histórico en el que se desarrollan estas mujeres. Así, las diferencias ideológicas y la inversión de valores en el mundo femenino y masculino se muestran latentes durante la Edad Media y el Siglo de Oro españoles. Ambos describen operaciones en las cuales el hombre y la mujer trabajan levemente mancomunados por la causalidad social e histórica, teniendo en cuenta la asignación de roles biológicos y sociales impuestos por el sistema imperante.

Sin embargo, Lorna Polo-Alvarado da la estocada final en el capítulo seis "Las mujeres en las ciencias" donde presenta avances de la historiografía literaria en la cual se estudia la participación de las mujeres en los saberes científicos en cuanto a salud, ciencias naturales, entre otros. Los estudios historiográficos contribuyeron al reconocimiento de las mujeres en los distintos estratos sociales, cuando en realidad fueron vedadas y omitidas voluntariamente por el poder masculino. Aunque la investigadora menciona varias mujeres con habilidades científicas, sobre todo, durante la Revolución Científica de los siglos XVI y XVII, todavía a las mujeres se les consideraba con valores duales, donde el poder y la destrucción se amalgamaban de tal manera que atentaba contra la naturaleza $^{13}$. Desde el principio la mujer tuvo que luchar contra los poderes

12 Lorna Polo-Alvarado, Vida de mujeres: entre la sumisión, 106.

13 Lorna Polo-Alvarado, Vida de mujeres: entre la sumisión, 129. 
que la cercaban para poder acceder a las ciencias, y muchas veces hasta los ejercía ilegalmente para poder involucrarse en decisiones sociales y de salud.

Por último, una pregunta llega al caso en la presente reseña: ¿Con qué propósito se escribe un libro hoy en día cuyo tema es la vida de mujeres en la antigüedad? ¿Qué se espera con la lectura y análisis de dicho libro? La respuesta la tiene ILBA (International Latino Book Award), cuyas premiaciones se han convertido en uno de los más grandes reconocimientos culturales a los latinos en los Estados Unidos. El libro en cuestión Vida de mujeres: entre la sumisión y la subversión, escrito por la Dra. Lorna Polo-Alvarado, obtuvo el segundo lugar en las categorías Best History Book - Spanish y en Best Women's Issues Book en Los Ángeles, California el 21 de septiembre del presente año. Este logro lo alcanzó entre 2,893 autores compitiendo, 257 finalistas en 95 categorías. Igualmente, Lorna Polo-Alvarado ha sido reconocida en su tierra natal Puerto Rico como también en otros países por su trayectoria académica como escritora, ensayista, conferencista y profesora de aula. Sus ponencias toman varios matices desde la literatura, el pensamiento crítico, la cultura y la escritura. Su fama como literata la ha llevado a dictar seminarios internacionales en Estados Unidos, Túnez, Alemania, Cuba, Panamá, República Dominicana, México y Colombia, entre otros.

ILBA reconoció no solamente la trayectoria de la investigadora Polo-Alvarado sino su producción literaria y la contribución que hizo acertadamente para el estudio de las mujeres en la antigüedad. Esto responde a la pregunta anterior puesto pone la premiación como reacción ante las demás entregas de producción historiográfica. La aproximación literaria de Lorna Polo-Alvarado es un llamado a los lectores e investigadores contemporáneos a considerar la vida de las mujeres desde la dimensión cultural, estética y académica, no sin descuidar su importancia en las ciencias sociales y en las humanidades. El texto historiográfico Vida de mujeres abarca la combinación de tradiciones académicas e históricas que las mismas mujeres de la antigüedad encarnaron. Esta investigación aporta un campo abierto a las diferentes disciplinas del conocimiento humanístico y filosófico originados en continuas conversaciones dentro de diversos saberes culturales e históricos. Igualmente, Vida de mujeres busca aclarar y definir objetos y sujetos de estudios aparentemente olvidados para proclamar un nuevo concepto en el proceso literario. 
El texto Vida de mujeres: entre la sumisión y la subversión establece un controvertido, pero aliciente discurso sobre lo femenino mal interpretado durante muchas épocas a propósito del abuso de poder masculino tanto de emperadores, reyes, sacerdotes, religiosos y críticos literarios que han cercenado la verdad durante siglos. Lorna Polo-Alvarado escribe con una prosa clara y emblemática posicionando su investigación entre las fuentes historiográficas más importantes que los profesores universitarios podemos echar mano para cambiar mentalidades y comportamientos en los estudiantes. En términos del texto y contexto del presente libro, podría considerarse una de las más importantes investigaciones de un crítico contemporáneo sobre la vida de las mujeres de la antigüedad. La emergencia de la vida femenina ilustra una periferia historiográfica totalmente diferente a la que se había venido desarrollando hoy en día. Aunque el texto de Polo-Alvarado es académico en cuanto la investigación y la escritura, propongo utilizarlo como recurso pedagógico en las clases de literatura o de humanidades en los programas de español en universidades extranjeras. Cada capítulo se ha tejido con destreza, naturalidad y sencillez que el texto provee una orientación clara y discursiva en su narrativa de mujeres.

Finalmente, el libro de la profesora Lorna Polo-Alvarado es una excelente guía literaria para ávidos y curiosos lectores porque, aunque es un libro especializado, igualmente es presentado con una narración nada ampulosa ni complicada de entender. Más bien, los puntos de encuentro presentados en el libro benefician aun a aquellos que quieren leer con el ánimo de aprender algo diferente, algo nuevo, para desarrollar nuevos ángulos de conocimiento y penetrar mucho más en el mágico mundo de la literatura y la historia.

\author{
Alexander Steffanell \\ Lee University (Estados Unidos) \\ asteffanell@leeuniversity.edu
}

